\title{
An Application Oriented Short-Course in Image Recognition.
}

\author{
F.Tarrés, E.Bertrán, G. Montoro, A.B. de Souza. \\ Escola Universitària Politècnica del Baix Llobregat. \\ Universitat Politècnica de Catalunya. \\ C/ Generalitat s/n. 08960. Sant Just Desvern. Barcelona. \\ Telf: 34-3-401 5896. \\ Fax: 3434015910 . \\ e-mail:
}

\begin{abstract}
.
An experimental course on Digital Image Processing and Recognition, together with its associated, are described. The program is a user-friendly interface running under Matlab 4.0, which allows easy-control over the main stages of any optical recognition system. It is used as an aid to illustrate the development of a complete image recognition application in a short-course which is mainly supported by laboratory experiments. The course has been specifically oriented to non-initiated students.
\end{abstract}

\section{Introduction.}

Most courses on Image Recognition are focussed towards a global and application-independent perspective. Different methods and procedures for image conditioning, segmentation, representation and recognition are usually covered in detail, discussing its advantages, drawbacks and suitability for specific problems. Examples covering a wide range of applications are frequently considered, trying to give an adequate perspective to problem solving. The authors believe that this approach is accurate for a semester course since it provides enough insight into the area so as to prepare the student to solve real applications. Nevertheless, students must have a strong basis on mathematics, digital signal processing and programming that restricts the audience to advanced university courses.

The approach presented in this paper was originally designed as a 15 hours seminar on image recognition adapted to students of a three-year carrier on telecommunications. The aim of the seminar was double: (1) to offer a general perspective of the different stages involved in any automatic recognition system and (2) to develop a complete real application from image acquisition to data recognition. Although these students have a reasonable good knowledge on mathematics and signal processing, no previous knowledge assumptions were made in order to be able to extend the course to professionals, technicians and medium degree students. We consider that the advanced mathematics used in most books and courses of image processing [1-3] impede its access to medium degree students, professionals or technicians, which probably are not directly related with electrical engineering or computer science but that are connected, in any or other form, to the computer control or supervision processes. Therefore, this course is also intended to serve as a first introduction to these technicians, trying to give some perspective on what is and what is not possible to recognize with vision systems and, eventually, allow them to resolve some easy and well-posed problems. Clearly, when no previous knowledge on digital signal processing concepts is assumed, the course must be extended to about 50 hours to guarantee that the main ideas of digitalization, filtering or representation notions are well understood (at least at a simplified and intuitive level).

Next section gives a general overview of the problems addressed in the course. The characteristics of the software package are described in section III while the contents covered in each laboratory session are summarized in section IV. Finally, section V outlines the main conclusions of our experience.

\section{General overview of the course.}

The course is based on the definition and development of a well-known application: car license plate recognition for automatic user identification in parking lots and it relies on the direct computer experimentation of different procedures, schemes and 
alternatives that are explained during the classes. A user-friendly program, which runs under Matlab 4.0, is used as the main tool for the analysis and evaluation of pre-coded algorithms and the development of new ones. The soltware has been designed to be operated without any prior knowledge of Matlab. Nevertheless, it is an open system where the user can interact writing its own code in conventional Matlab. The idea is to begin with the image recognition problem without worrying about the programming language. As the course progresses, students are requested to write its own code for some simple procedures not incorporated in the package. Eventually, they will be able to understand the programming philosophy of Matlab and create their own programs using the resources of the Image Processing Toolbox. Obviously, when the course is imparted to professionals or students without experience in programming, the contents is relaxed and all the required procedures are included in the working package.

The problem of car license plate recognition was selected as the guiding experiment since it is an open but feasible application that involves different image processing areas such as image enhancement, identification of the region of interest, segmentation, information representation and description, recognition and classification. Furthermore, it is a highly motivating and attractive problem for the students.

The present version of the program includes general image processing routines together with specific programs that were specially designed for the license plate recognition problem [4]. However, as described in the next section, the structure of the menus and the user interface is adapted to solve any general image recognition problem and new procedures are currently being generated for addressing other well-known applications.

We are aware of the limitations of this approach that inevitably leads to a limited vision of the full area of optical recognition. Nevertheless, the original purpose was just to provide some elemental understanding of these topics in the curricula of a telecommunication engineer (or any other professional). Some of these students will continue their learning on this area in more advanced courses while others not. Experience reveals that the course is useful for both groups, as a motivating introduction for the first and as a useful professional aid for the later.

\section{Description of the image analysis and recognition software.}

As noted before, this program is used as the main learning tool and its structure and user interface have been optimized from an educational point of view. The package have been developed under Matlab 4.0 for a number of reasons comprising its extended use in the scientific community, easiness for programming, existence of dedicated image processing toolboxes, etc. Moreover, the final intention of this package is to progressively introduce the student on the programming principles of Matlab allowing them to develop their future experiments under a well-known commercial software.

The user interface resembles any standard windows application. It uses a total of eight curtain menus that are associated to conceptually different stages on the image recognition process. Additional menus implementing useful Windows functions such as Edit, View and Help are also implemented. The eight main menus are: File, Preprocessing (tuning), Region of Interest, Segmentation, Object Description, Database, Recognition and General Sequencing. Each menu is considered as a different module that performs different type of operations on the image. These modules are activated and controlled from the General Sequencing Menu. A brief description of the functions and procedures supported in each menu and an outline of the data transferring structure is given below.

File Menu: It is devoted to the input and output functions. The options Open, Close and Save are used for retrieving or saving the active image from or to disk. The active image is assigned to a user variable named act_imag that is always represented in a graphic window. The Open option can support any standard type file format, maintaining its original size but converting it tó 256 level black and white image. The Save option only works in the 256 level bitmap format.

The option Acquire controls an image acquisition board that is supposed to be connected to a camera. In practice the camera is replaced by a vidco signal from a VTR that constantly replays a previously registered sequence of cars entering a parking lot. The options Print and Exit are also included in this menu for hardcopy of the active image and quit the program. 
Preprocessing Menu (tunning): It contains several image enhancement routines: Gamma correction, Thresholding, Contrast Stretching, User defined point to point transformations, Averaging, Median Filtering, User defined filters, Equalization, Adaptive Equalization, Histogram Specification, Histogram analysis, etc. These procedures may be run in a batch or in an interactive manner. In the latter case, the program asks for the relevant procedure parameters and applies them to the active image. A sensitive help window may be to assist the user on the parameter selection. Up to 5 previous active images may be restored via the Undo option on the Edit Menu.

The Define Batch Sequence option allows the user to define a sequence of operations to be systematically applied to the image. This option is also included in other modules and is used for defining a repetitive sequence that is systematically applied to the image. The batch sequence is defined in a text window using a simple and intuitive Matlab inspired language. A text sequence like:

\section{Imag I=equalization(act_imag);}

act_imag=thresholding(Imagl, 25);

will equalize the histogram of the image (not presenting the result) and will apply a threshold of 25 to the resulting image (presenting the result at the graphic window). The sequence of the operations may be as large as desired and is activated through the Execute Batch option. Additional image results may be represented using the instruction:

Winfig_N=Represent(Imagl);

where Winfig_N represents the $\mathrm{N}$-thm graphic window. These windows may be closed using the command:

$$
\text { Close (Winfig_N); }
$$

The result of applying the image enhancement routines is passed to other modules via the act_imag variable. This variable is invariably updated when running the program in interactive mode. In batch processing, the user must settle this variable. The Matlab codes associated to the routines included in the Preprocessing Menu are stored in a specific directory. Users can add new routines to this menu writing the appropriate Matlab code. The program automatically examines these directories and rearranges the menus to combine all the procedures. The same scheme is used for generating new user-codes on the other modules of the program.

Region of Interest Menu: The aim of this module is to extract the significant information of the image to be further processed. In many recognition problems the region of interest is the complete image and this step should be omitted. In our application, it consists on selecting the rectangular region of the plate from the car and the surroundings. Usually, the extraction of the region of interest is a highly applicationdependant procedure.

Our implementation consists on analyzing the contents of different lines of the image and determining if the line belongs to the license plate or not. The analysis is based on applying a binary threshold to the candidate line and examining the number of occurrences of different parameters. The user may control the number of black or white pixels, the ratio of black vs. white pixels in the line, the number of transitions, the number of pixels in transitions among black and white or viceversa, etc; and can define a condition to establish if the line belongs to the plate. The criteria for selecting the lines may be random or guided. Binarization may be carried out via a fixed or a dinamic threshold [3]. The analysis is repeated until a candidate line is detected.

After line detection, a region growing procedure, based on the gray level of the background, is used to find the image region containing the license plate. The region-growing algorithm begins at the central point of the line and extends uniformly in four directions. The user can control the gray level condition and the selection of pixels for region growing. Once the region of interest has been found act_imag will only contain the subimage corresponding to the license plate.

Segmentation Menu: The segmentation menu contains a number of procedures for extracting the characters of the license plate. Different alternatives and parameters may be selected. The analysis begins examining the columns of the image that are candidate to contain a character. The procedure is similar to the one used in the Region of Interest Menu but it works with different conditions. Once the appropriate column has been detected, the character is extracted via a region growing algorithm. General-purpose morphological operators are also included in this menu in order to further process each character. As in other modules, the user can control the segmentation process in an interactive or batch manner. The results of the segmentation are transferred to other modules via an array of images.

Object Representation Menu: In the actual version the description of the digits is made via projection of 
the image in different directions. A normalization procedure is applied to the characters before implementing the projection. Users can select the number of projections and its directions. Projected functions of prefixed dimensions are stored in a matrix for transferring the information to the Database Construction or Recognition Menus.

Database Menu: It is used for interactive or batch processing object description information in order to obtain representative statistical functions of each character. It is an optional module since previous generated databases are supplied. User can generate its own database or select one of the pre-defined. The representative of each character is obtained as the mean values of the functions describing its different realizations.

Recognition Menu: Provides control over the distances between the current character description functions and the database representatives. Distance computation may be correlation, absolute difference or quadratic error. Threshold values for acceptance or non-acceptance of a character can also be adjusted.

General Sequencing Menu: It provides control over the complete sequence of algorithms applied to the image. The instructions are given in a text window and the strategy of programming is very similar to the Define Batch cption included in the other modules. As an example, we consider the following sequence:

File < Acquire; $>$;

Preprocessing;

RegionOfInterest;

Preprocessing

$<$ act_imag=Threshold(act_imag,100); >

In this example the image will be loaded from the VTR and the preprocessing instructions specified at the Define Batch option will be applied. After region of interest detection the image is binarized with a fixed threshold of 100 . As indicated in this example, specifying a sequence of operations after the module call inhibits the execution of the batch queue. Loop control instructions such as for, do while, etc, are also allowed.

\section{Course contents.}

The course imparted to telecommunication engineering students is divided in 10 laboratory sessions of $1 \mathrm{~h} 30 \mathrm{~min}$. The version for medium degree students or professionals takes about 5 hours per session. A brief outline of the contents is given below:

S1: Introduction. Image Acquisition. Image File types. Opening and representing files with Matlab. General steps on a Recognition problem.

S2-S3: Image enhancement. Description and test of routines included in the package. Development of new user-defined routines.

S4-S5: Searching the region of Interest. Test and develop procedures.

S6: Segmentation: basic concepts. Test and develop procedures.

S7-S8: Character description: The method of projection. Test results with different segmentation alternatives.

S9-S10: Evaluate recognition capabilities of the system and discussion of other possible alternatives to solve the problem [5].

\section{Conclusions.}

The course effectively introduces fundamental concepts of Image Recognition via experimentation with a highly motivating application. Students get the sensation that real systems are feasible and are ready to continue their research, supervised or not, with more advanced methods and different applications.

\section{Bibliography.}

[1] R.C. González, R.E. Woods. "Digital Image Processing". Addisson Wesley. 1987.

[2] A.K. Jain. " Fundamentals of Digital Image Processing”. Prentice Hall. 1989.

[3] J.M. White, G.D. Rohrer. "Image Thresholding for Optical Character Recognition and other applications requiring Character Image Extraction". IBM J. Research and Develop. Vol 27. No 4. Pp 400-406. July 1983.

[4] A.B. de Souza. "Reconocimiento Optico de Matrículas". TFC. EUPBL.Sept. 1997.

[5] M.S. Suen; C.Y. Yamamoto. "Historical review of OCR research and development". Proc. IEEE. Vol 80, 7, July 1992. 